\title{
Análisis de la reducción del consumo de ancho de banda de un ISP mediante el estudio de Servidor Web Proxy Cache
}

\author{
DOI: https://doi.org/10.33262/ap.v3i3.2.112
}

(c) (1) ()(2)

Analysis of the reduction of the bandwidth consumption of an ISP through the study of the Web Proxy Cache Server

\author{
Marco Vinicio Ramos Valencia. ${ }^{1}$, Steven Alejandro Salazar Cazco. ${ }^{2}$, Jhonatan \\ Guillermo Díaz León. ${ }^{3} \&$ José Alberto Rivadeneira Romero. ${ }^{4}$
}

\begin{abstract}
Introduction. Bandwidth consumption is a valuable resource in companies today, the need to constantly improve response times in requests for web services has led to the search for efficient means that meet the requirements of the network, decreasing the bandwidth that is consumed. Objective. Analyze the reduction of the bandwidth consumption of an ISP by studying the Web Proxy Cache Server for the company FASTNET CIA. LTDA. Methodology. The research design was quantitative. An analysis of the TELCO network was carried out applying the Comprehensive Methodology for Advanced Networks (MIRA), thus obtaining its strengths and weaknesses. Consequently, different cache proxy web server options were studied, including: HAARPCACHE, RAPTOR CACHE, SQUID, THUNDERCACHE, expanding the range of options for proper implementation. three scenarios were configured; the first base its structure on the node "Nat Langos AS" without any variant, the second scenario was differentiated by having the HAARPCACHE server installed on the HP Proliant dl-385P server and the third changes the server for RAPTOR CACHE

\footnotetext{
${ }^{1}$ Escuela Superior Politécnica de Chimborazo, Facultad de Informática y Electrónica. Riobamba, Ecuador. vi_ramos@espoch.edu.ec, https://orcid.org/0000-0003-3033-2404

${ }^{2}$ Escuela Superior Politécnica de Chimborazo, Facultad de Informática y Electrónica. Riobamba, Ecuador. steven.salazar@espoch.edu.ec, https://orcid.org/0000-0002-9708-5885

3 Investigador Independiente, Riobamba, Ecuador. jhonatan_diazl@outlook.com https://orcid.org/00000003-1888-9605

${ }^{4}$ Investigador Independiente, Riobamba, Ecuador. alberto_rr@outlook.com https://orcid.org/0000-00033128-7975
} 
implemented in it. computer. Results. The HAARPCACHE server met $90 \%$ of these conditions and resulted in $17.25 \%$ savings in business bandwidth, while RAPTORCACHE met $45 \%$ of the measures and created savings corresponding to $8.82 \%$. Conclusions. It is concluded that the most suitable server for FASTNET CIA. LTDA. Due to its reliability and saving capacity, it is HAARPCACHE, in addition to a considerable reduction in bandwidth, thus improving user performance.

Keywords: Web server, proxy, cache, internet, MIRA, bandwidth.

\section{Resumen}

Introducción, el consumo de ancho de banda es un recurso valioso en las empresas hoy en día, la necesidad de mejorar constantemente los tiempos de respuesta en las solicitudes de los servicios web ha provocado que se busquen medios eficientes que cumplan con los requerimientos de la red, disminuyendo el ancho de banda que se consume. Objetivo, analizar la reducción del consumo de ancho de banda de un ISP mediante el estudio de Servidor Web Proxy Cache para la empresa FASTNET CIA. LTDA. Metodología, el diseño de la investigación fue cuantitativa. Se realizó un análisis de la red TELCO aplicando la Metodología Integral para Redes Avanzadas (MIRA) obteniendo así sus fortalezas y debilidades. Consecuentemente se estudiaron diferentes opciones de servidores web proxy caché, entre ellos: HAARPCACHE, RAPTOR CACHE, SQUID, THUNDERCACHE, ampliando el abanico de opciones para una implementación adecuada. se configuraron tres escenarios; el primero basa su estructura en el nodo "Nat Langos AS" sin ninguna variante, el segundo escenario se diferenció por tener incorporado el servidor HAARPCACHE instalado sobre el servidor HP Proliant dl-385P y el tercero cambia el servidor por RAPTOR CACHE implementado en el mismo ordenador. Resultados, el servidor HAARPCACHE cumplió en un 90\% de dichas condiciones y provocó un 17,86\% de ahorro de ancho de banda empresarial, mientras que RAPTORCACHE cumplió con el $45 \%$ de las medidas y creó un ahorro correspondiente al 8,92\%. Conclusiones, se concluye que el servidor más adecuado para FASTNET CIA. LTDA. por su fiabilidad y capacidad de ahorro es HAARPCACHE, además de una considerable reducción del ancho de banda mejorando así el performance de los usuarios.

Palabras claves: Servidor web, proxy, caché, internet, MIRA, ancho de banda,

\section{Introducción}

En la actualidad, el Ecuador no cuenta con empresas nacionales que se dediquen a esta actividad específicamente, sin embargo, podemos encontrar empresas extranjeras que ofrecen este tipo de servicio, como por ejemplo la empresa THUNDERCACHE de Brasil, que se dedica principalmente a dar soluciones sobre los consumos de ancho de banda para quienes lo requieran. 
En la presente investigación se implementarán escenarios de estudio en los que se incluye servidores web proxy caché, de los cuales se medirán los niveles de consumo de ancho de banda para una posterior evaluación. FASTNET Cía. Ltda. contará con un sistema de cacheo web de bajo costo de implementación y un alto grado de eficiencia, con el objetivo de reducir el consumo de ancho de banda en la red y principalmente mejorar los tiempos de respuesta.

Al mejorar los tiempos de respuesta en las solicitudes de los servicios web ha permitido que se busquen medios eficientes que cumplan con los requerimientos de la red. Además, reduciendo el ancho de banda que se consume. El servidor web proxy cache permite llevar a cabo dicha optimización disminuyendo en gran cantidad el consumo de ancho de banda, ya que se puede tener de forma local los recursos que se solicitan a un sitio web (Rodríguez, 2015).

En el presente artículo se propone una medida que contiene la implementación de un servidor web proxy cache, el control que ejercerá este sobre el acceso y salida de solicitudes, son pensadas a favor de la mejora de ancho de banda, el estudio se enfoca en la empresa FASNET. Cía. Ltda.

El incremento del uso de las redes datos, su estancia a través del tiempo y su agresiva capacidad de introducirse como una necesidad han proporcionado el ambiente perfecto para que se muestren dificultades y obstáculos para el eficiente uso de los recursos que participan en la conectividad, al mismo tiempo la habilidad propia del ser humano por solucionar problemas, surgen nuevas y mejores posibilidades con el objetivo de optimizar las redes (Jordan, 2019).

Durante la investigación nos hemos encontrado con varias herramientas, como por ejemplo la implementación de servidores, metodología que brinda una organización para la impaciente demanda de conectividad y como puerta de escape a problemáticas de las empresas.

\section{Metodología Mira}

Se define como producto del perfeccionamiento de aplicaciones de proyectos que se enfocan en la caracterización y el análisis de las redes. Esta metodología no sólo permite un análisis por paquetes, sino que también ahonda el estudio en el contenido, esta metodología a través de sus mecanismos definidos ofrece la oportunidad de entender el comportamiento actual de la red y pronosticar la conducta de la misma en el futuro (Cayo, 2015).

Esta metodología al permitir una configuración individual de cada módulo es una ventaja de MIRA como muestra la figura 1. 


\section{Figura 1}

Arquitectura Funcional Mira

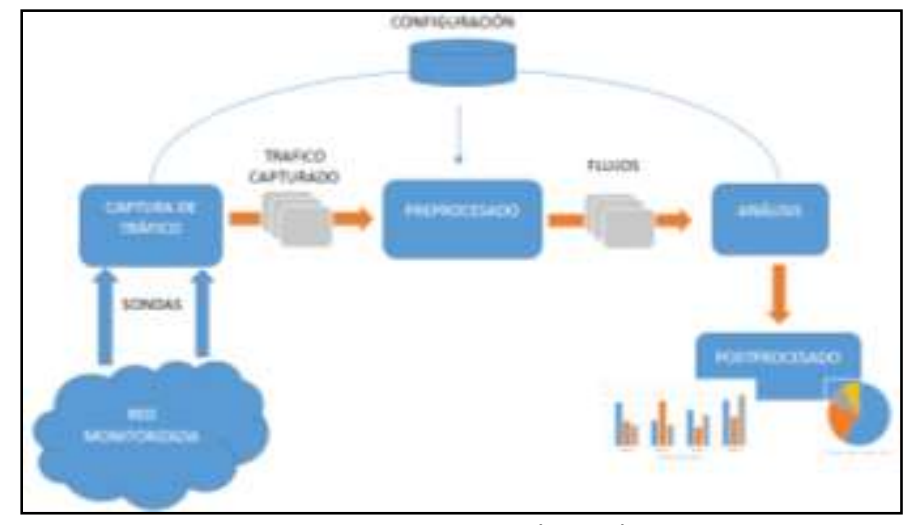

Fuente: Diaz y Rivadeneira (2015)

Otra de las ventajas de esta metodología es su adaptabilidad a distintas tecnologías; 802.13, 802.11, etc. (Romereal, 2020).

Mira ofrece cuatro módulos:

- Módulo de Captura. - Comprende la captura del tráfico a través de una herramienta de software apropiada y acorde a la tecnología sobre la cual se pretende realizar el monitoreo.

- Módulo de Preprocesado. - A través de un filtro establece el nuevo contenido que se desea manejar. La correlación entre el tráfico de un sentido y de otro nos da como resultado la obtención de un flujo bidireccional (biflujo) enlistado con sus respectivos síntomas para cada sentido (Verciana, 2019).

- Módulo de Análisis. - Realiza un cruce de atributos con los datos preprocesados así el resultado brinda informes finales de gran utilidad para el analizador.

- Módulo de Integración. - Compacta más la información y dependerá de los módulos de análisis que sean creados.

\section{Tipos de servidores}

Con el boom del internet se desarrolló soluciones de carácter informático con el objetivo de mejorar su rendimiento, mediante las cuales han aparecido los servidores que hoy en día son considerados como uno de las mejores herramientas desarrolladas para que sean implementadas para la red.

Servidores Proxy. - Éste es un servidor HTTP especial, su funcionamiento consiste en esperar una petición de los clientes de la red, posterior la procesa y envía una solicitud hacia el servidor remoto que se encuentra fuera de la red del cliente, procede a leer la respuesta que llega al servidor de tipo proxy y retorna al cliente que realizó el pedido.

Como los servidores proxy se encuentran de intermediarios entre la subred e Internet, es una ventaja para el cacheo de datos ya que todas las peticiones deberán pasar a través de él. 
Los servidores proxy tenemos dos tipos de proxy correctamente establecidos:

- Circuito. - Estos tipos de proxies también conocidos como genéricos, básicamente son una conexión virtual entre host cliente y el host servidor.

- Aplicación. - Son aquellos que están configurados para permitir o denegar el acceso a una determinada aplicación, consiste en que el servidor entenderá e interpretará tan solo al protocolo de la aplicación, también son conocidos como servidores proxy dedicados (Altis \& Luotonen, 2014).

Servidores Cache. - Tienen la función de almacenar contenido (paginas HTML, imágenes, documentos, entre otros.) de los sitios web visitados por los usuarios para acceder a ellos de forma rápida, optimizando así el rendimiento de un sistema o de una red.

Existen tres tipos de caches que atienden las solicitudes de un sitio web:

- De Agente de Usuario: Solo funcionan para un usuario, se encuentra en los navegadores web.

- Compartida: Tiene como fin reducir el ancho de banda consumido, ya que todos los usuarios que acceden a internet comparten el mismo recurso, es el tipo de cacheo más común entre los ISP y empresas,

- Pasarela: Este tipo de caché funciona como un servicio de respaldo para un servidor web, de forma totalmente transparente para los clientes.

Los servidores web proxy cache son aquellos elementos situados al interior de una LAN (red local), aquí llegan todas las peticiones del servicio web (HTTP, HTTPS) que realiza el usuario en lugar de alcanzar el servidor de origen, parte del contenido solicitado es alojado en la memoria del servidor para un próximo pedido (Galarza, 2015).

Servidor Thundercache. - Es de alto rendimiento, optimizado para operar en entornos de alto tráfico tazas de transmisión de $1 \mathrm{~GB} / \mathrm{s}$ en un mismo servidor. Este posee un resumen de descargas, en interrupciones de descargas, el servidor continuará siendo la misma en el punto que se interrumpió. Además, no depende de Plugins, los Plugins obsoletos o necesarios para ciertas páginas son descartados o actualizados de forma automática por el servidor (Costas, 2015).

Servidor Raptorcache. - Posee sistema proxy cache caracterizado por almacenar contenido dinámico, pude guardar distintos tipos de contenidos cuando se navega. Al realizar las peticiones nuevamente los objetos solicitados son entregados desde el servidor, lo cual produce un ahorro de ancho de banda de internet a una mayor velocidad mejorando el servicio sustancialmente (Mikrocaché, 2020).

Servidor Haarpcache. - Servidor creado en licencia GPL V2, servicio desarrollado para el cacheo de contenido de forma dinámica, crea conexión cliente servidor Haarpcache y contesta las solicitudes mostrando el contenido solicitado, si el contenido no es hallado en el servidor se procede a dar paso a la solicitud hacia el servidor donde se origina la 
información. Mejora la velocidad en que se muestra el contenido y hace eficiente el uso del recurso de ancho de banda (Haarpcache,2018).

Servidor Squid. - Es un proxy de cacheo para el soporte de protocolos como HTTP, HTTPS, FTP, y más. Reduce el consumo de ancho de banda y el tiempo de respuesta mediante el cacheo y reutilización de peticiones frecuentemente efectuadas. Squid posee controles de acceso extendidos y crea un gran servicio de aceleración. Puede ser utilizado bajo cualquier sistema operativo (Squid Cache Org.,2018).

\section{Topología}

La red de datos de Fastnet CÍA. LTDA. utiliza una topología de tipo jerárquica o de árbol y está divida en 6 nodos principales, en los cuales se encuentran la red interna como también las redes de acceso y distribución, todas estructuradas de forma que logran la cobertura total de su servicio, como muestra en la figura 2.

\section{Figura 2}

Topología general de la red de Fastnet Cía. Ltda.

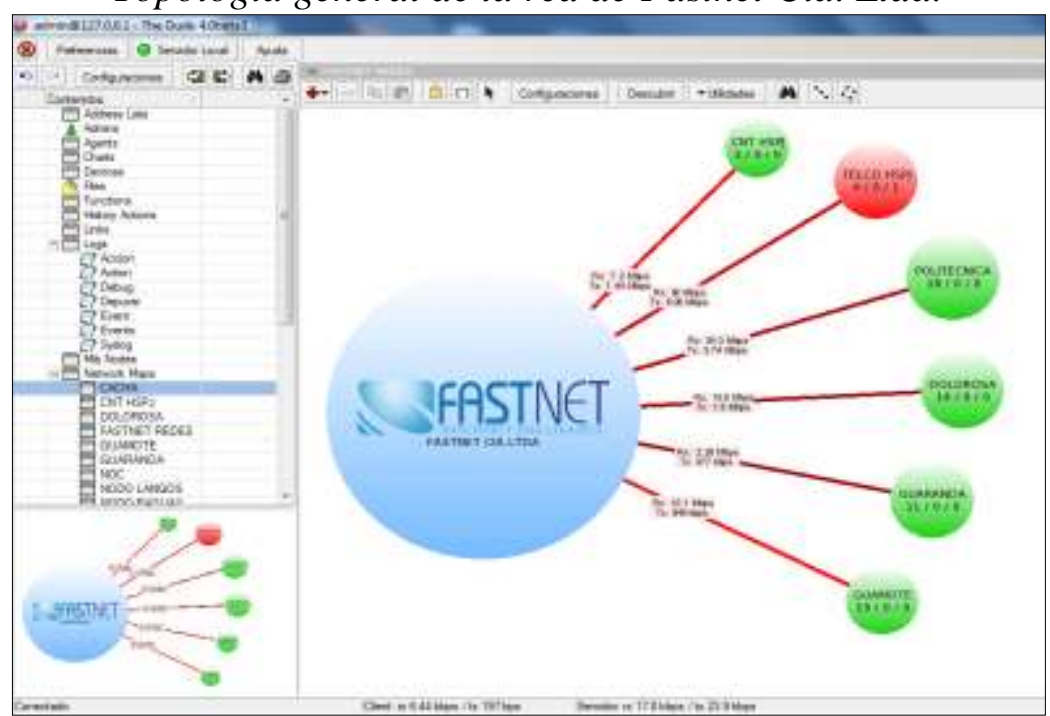

Fuente: Diaz y Rivadeneira (2015)

La configuración de los nodos principales está establecida bajo la misma premisa de la topología de jerarquías mencionada anteriormente, algunos nodos se dividen en secundarios o subnodos que mantienen la rediviva estructura, los cuales son: CNT HSPJ, TELCO HSPJ, POLITÉCNICA, DOLOROSA, GUARANDA, GUAMOTE. Dentro de su extensa red de cobertura para el servicio de internet cuenta con 922 clientes, distribuidos de la siguiente forma: CNT HSPJ 34, TELCO HSPJ 436, POLITÉCNICA276, DOLOROSA 129, GUARANDA 18 y GUAMOTE 29

\section{Ancho de Banda}

El mecanismo indicado para determinar la cantidad de información transmitida dentro de un segundo a través de un canal o medio de transmisión es con el término ancho de banda. Esta definición ha sido mal utilizada, debido a que es empleado únicamente como base indirecta para el cálculo de la capacidad del medio. Su unidad de medida es en Hertz 
mientras que los bits por segundo (bps) es la unidad de la tasa de transmisión (González, 2014).

Cuando con referimos al ancho de banda hablamos de un recurso de mayor importancia de la transmisión de datos a través de la Internet, comúnmente manejado por los ISPs para catalogar los planes que estos proveen, la creciente demanda de servicios por los usuarios ha provocado un gran aumento del consumo de tal recurso, estudios revelan que para el año 2018 se observará que el consumo más alto de banda ancha en el mundo se quintuplicará (Díaz y Rivadeneira, 2015).

La empresa FASTNET CIA. LTDA. tiene un contrato directo con dos ISPs en el Ecuador: la Corporación Nacional de Telecomunicaciones (CNT) y TELCONET, mismos que proveen a la empresa con un recurso de ancho de banda de $20 \mathrm{Mbps}$ y $156 \mathrm{Mbps}$ respectivamente. El ancho de banda se encuentra distribuido en planes establecidos para la prestación de su servicio de internet a la comunidad como se muestran en la figura 3.

Figura 3

Topología Backbone: ISP TELCONET / CNT

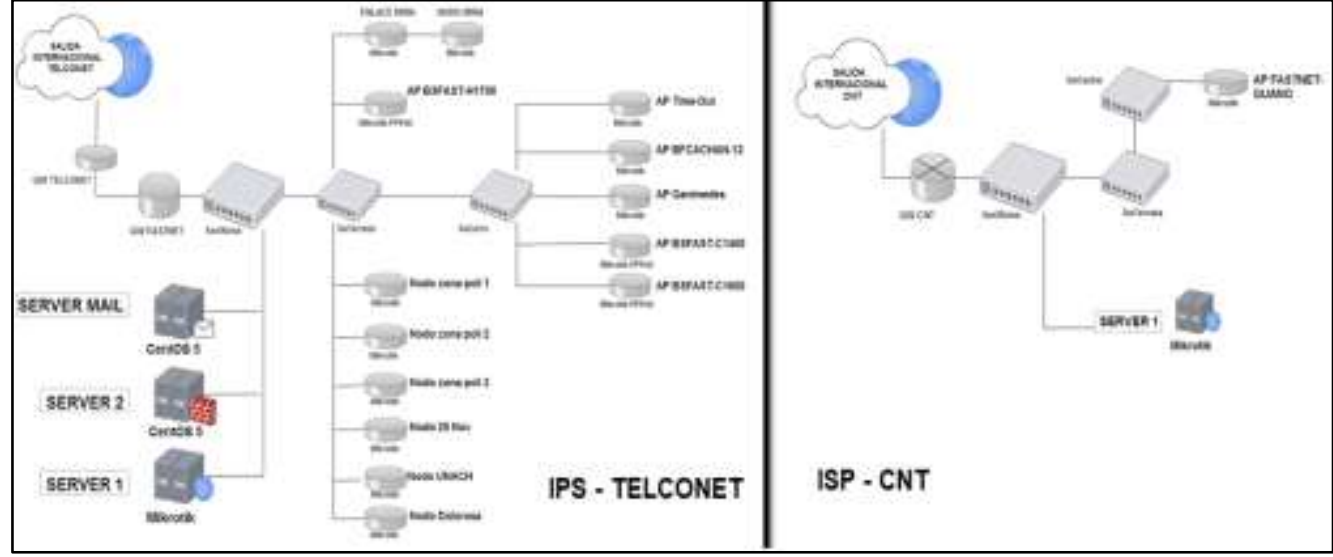

Fuente: Diaz y Rivadeneira (2015)

El ancho de banda total con el que la empresa cuenta es de $176 \mathrm{Mbps}$, este recurso se encuentra distribuido para sus seis nodos principales como lo muestra la Tabla 1 a continuación.

Tabla 1

Distribución ancho de banda por nodos principales

\begin{tabular}{cc}
\hline NODO PRINCIPAL & ANCHO DE BANDA [Mbps] \\
\hline CNT HSPJ & 4 \\
\hline TELCO HSPJ & 75 \\
\hline POLITECNICA & 53 \\
\hline DOLOROSA & 28 \\
\hline GUARANDA & 3 \\
\hline GUAMOTE & 13 \\
\hline Fuente: Diaz y Rivadeneira (2015) &
\end{tabular}

El nombre de cada nodo es asignado en función de la ubicación de la infraestructura principal del mismo. En algunos casos con referencia a lugares cercanos del nodo. 


\section{Metodología}

Para dar cumplimiento al objetivo de nuestro artículo planteado, la investigación se desarrolló utilizando la orientación cuantitativa mediante la recolección de datos con muestras y mediciones específicas de la red a ser analizada. Además, se debe tener en cuenta que la comparativa se realizará en un escenario simulado basado en el escenario real y los datos obtenidos serán comparados con el mejor rendimiento mostrado reflejado en la reducción del agotamiento de ancho de banda.

Para lograr un estudio adecuado del ancho de banda nos basaremos en tres parámetros: Escenarios, Análisis de Trafico y un pre - análisis de estos para luego poder compararlos con los resultados obtenidos con la inclusión de los servidores proxy cache.

\section{Escenarios}

Las redes de datos son topologías que se transmiten sus datos de una manera no guiada $u$ organizada, la mala configuración de recurso a la red puede generar consecuencias graves sobre el rendimiento de la red, el presente artículo es indispensable realizar una adecuada delimitación del área de estudio.

En la Tabla 2, la designación del nodo para el caso de estudio es asignada por el jefe técnico, para este caso es el subnodo NAT LANGOS AS mismo que se encuentra ubicado en el nodo TELCO HSPJ, cuenta con un ancho de banda teórico de 24Mbps y 89 clientes.

Tabla 2

Número de Clientes Nodo NAT LANGOS AS

\begin{tabular}{cccc}
\hline NODO & Nro. CLIENTES & UPLOAD & DOWNLOAD \\
\hline & 54 & $1 \mathrm{M}$ & $1 \mathrm{M}$ \\
\cline { 2 - 4 } NAT LANGOS AS & 1 & $1 \mathrm{M}$ & $2 \mathrm{M}$ \\
\cline { 2 - 4 } & 3 & $2 \mathrm{M}$ & $1 \mathrm{M}$ \\
\cline { 2 - 4 } & 30 & $2 \mathrm{M}$ & $2 \mathrm{M}$ \\
\hline
\end{tabular}

Fuente: Diaz y Rivadeneira (2015).

A continuación, como se muestra en la figura 4, 5 y 6 se describen los siguientes tres escenarios a ser utilizados para el estudio comparativo de los servidores:

Figura 4

Topología Escenario 1

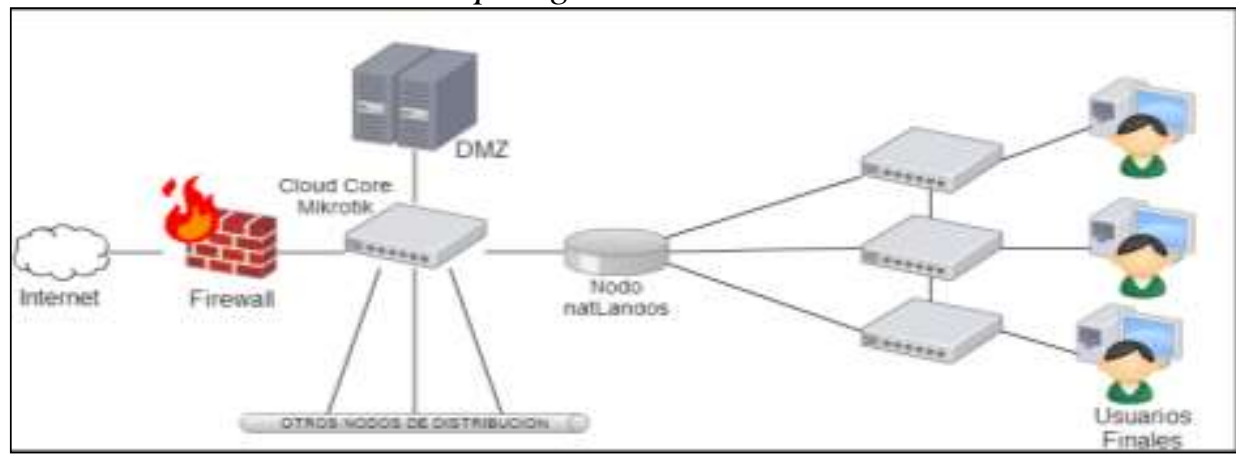

Fuente: Diaz y Rivadeneira (2015) 

septiembre, 2021

- Escenario 1 - Red Actual. - Figura 4, este fue la designado por el equipo del departamento técnico de la empresa, no se realizó ninguna manipulación ni variación con la finalidad de hacer un estudio íntegro sobre la red, así conocer las debilidades actuales.

- Escenario 2: Red Servidor 1.- El segundo escenario propuesto se encuentra definido sobre la misma red NAT LANGOS AS con la integración adicional del primer servidor (HAARPCACHE).

\section{Figura 5}

Topología Escenario 2

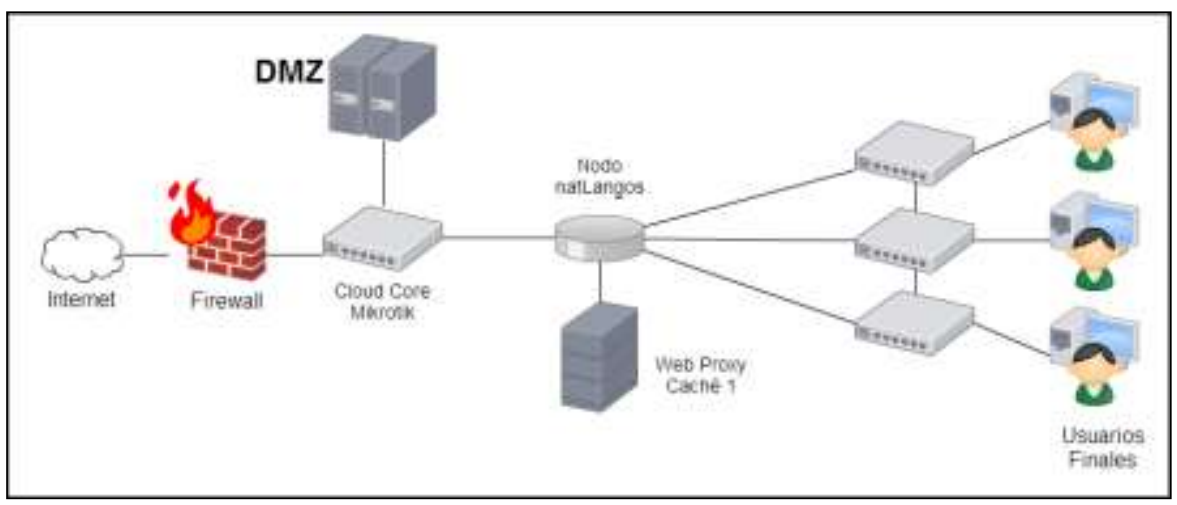

Fuente: Diaz y Rivadeneira (2015)

- Escenario 3: Red Servidor 2.- Por último, el tercer escenario planteado mantiene su establecimiento sobre la red NAT LANGOS AS, a diferencia del segundo escenario éste contará con un servidor web cache distinto, servidor 2 (RaptorCache)

Figura 6

Topología Escenario 3

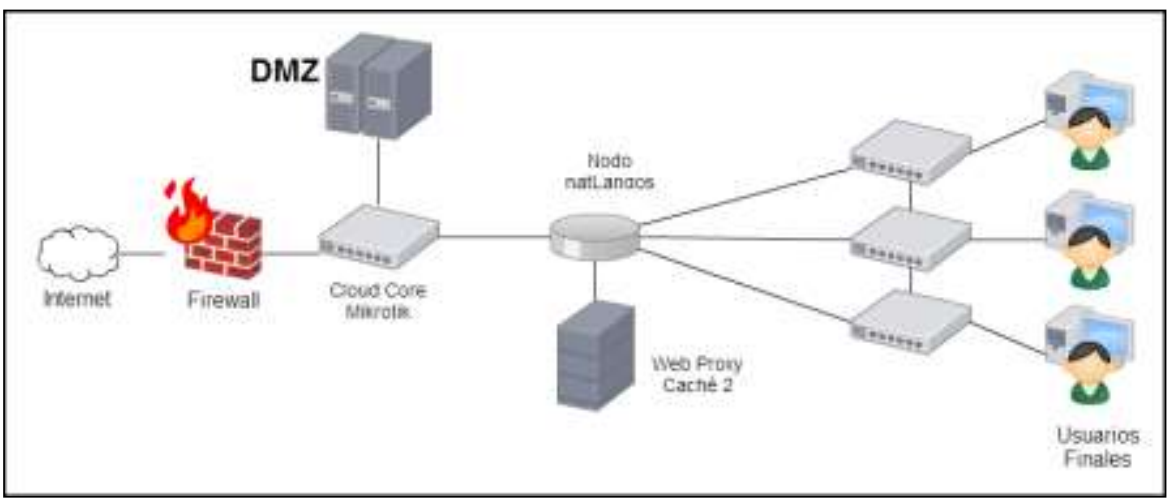

Fuente: Diaz y Rivadeneira (2015)

\section{Análisis de Trafico de los Escenarios Propuestos}

El consumo promedio del ancho de banda en la red TELCO (conjunto de nodos principales: TELCO HSPJ, POLITECNICA y DOLOROSA.) es de 135,29 Mbps al día, lo cual representa un $86.72 \%$ del total asignado a los Nodos Principales dentro de la ciudad 
El ancho de banda teórico consumido es de $97.5 \mathrm{Mbps}$, la asignación del recurso se establece adicionándole al teórico consumido el $60 \%$ del mismo con la finalidad de crear una proyección futura que permita el aumento del consumo del recurso con un correcto abastecimiento y distribución (Díaz y Rivadeneira, 2015).

Realizando el mismo cálculo, con un total de 841 clientes obtenemos el siguiente dato:

$$
\begin{gathered}
\mathrm{T}_{\mathrm{BW}}=\sum \text { plan } *(\text { clientes } / \text { partición }) \\
T_{B W}=1(512 / 8)+2(326 / 8)+4(3 / 8)=147 \mathrm{Mbps}
\end{gathered}
$$

El cálculo arroja un resultado de $147 \mathrm{Mbps}$ correspondiente al consumo de ancho de banda que en teoría debería consumir la red TELCO, si observamos, este resultado es muy próximo a los 135,29 Mbps hallados para el consumo promedio real (Montoya, 2019).

\section{Actividades de pre - análisis}

Para aplicar el estudio de tráfico sobre redes de gran número de usuarios resulta indispensable enfocarlo sobre una muestra debido a que los paquetes transmitidos pueden llegar a ser millones convirtiendo la tarea en algo imposible incluso con software y hardware de alta capacidad de análisis, bajo esta premisa se realizó una observación sobre el ancho de banda consumido por la red diariamente como lo muestra la Figura 7., la observación fue efectuada durante una semana. Los datos arrojados indican una frecuencia de mayor uso en los rangos de tiempo de $12 \mathrm{~h} 00$ a $17 \mathrm{~h} 00$ y de $20 \mathrm{~h} 00$ a $24 \mathrm{~h} 00$.

Los lapsos de tiempo escogidos para las capturas son: de $9 \mathrm{~h} 00$ a $9 \mathrm{~h} 10$, de $12 \mathrm{~h} 00$ a $12 \mathrm{~h} 10$, de $16 \mathrm{~h} 50$ a $17 \mathrm{~h} 00$ y de $21 \mathrm{~h} 00$ a $21 \mathrm{~h} 10$, estos tiempos corresponden a los días de revisión pre - mantenimiento y mantenimiento establecidos en el cronograma mensual de la empresa.

\section{Figura 7}

Consumo Ancho de Banda NAT LANGOS AS

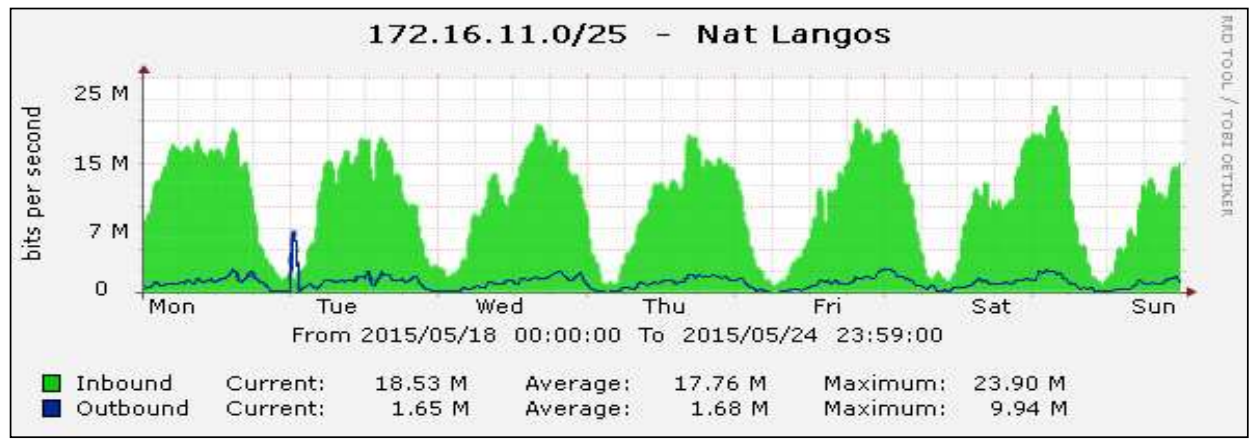

Fuente: Diaz y Rivadeneira (2015)

Uno de los requerimientos es el uso de licenciamiento libre, por tal razón el sniffer para nuestra red, teniendo en cuenta la generación de reportes, es Wireshark que además su 
interfaz es sencilla y fácil de manipular. Por lo tanto, el sniffer escogido fue WIRESHARK.

FASTNET CIA. LTDA. posee herramientas de software para la administración de dispositivos de red, entre las de mayor utilidad están WinBox y Dude, con las cuales la tarea se realiza de forma prolija y sencilla. WinBox permite una conexión virtual y remota con todos los dispositivos MikroTik que funcionan bajo RouterOS (Díaz y Rivadeneira, 2015).

Una alternativa apropiada para las fases del análisis de tráfico es realizar una sesión de monitoreo mediante la configuración de port mirroring. El puerto espejo o port mirroring en inglés, es una utilidad para redes de datos aplicado en dispositivos de switcheo para capturar el tráfico entrante y saliente del puerto elegido hacia una base de datos externo, con la finalidad de analizar dicho tráfico sin intervenir con el funcionamiento normal de la red (Mikrotik, 2020).

\section{Resultados}

\section{Análisis Mira Escenario 1}

La captura se la realizó sobre el dispositivo ROUTER BOARD 1100 X2AH en los lapsos de tiempo ya escogidos en la etapa de preparación previa al análisis, la red NAT LANGOS. Las capturas se realizaron en dos horarios durante dos meses:

- Realizado Físicamente: 09:00 - 09:10 y 12:00 - 12:10

- Realizado Remotamente 16:50 - 17:00 y 21:00 - 21:10

En promedio 600000 paquetes fueron obtenidos por cada sesión de captura, para un correcto manejo de estos datos se realizó una reducción inicial del tráfico capturado a través de filtros con la finalidad de eliminar información innecesaria para el estudio.

\section{Tabla 3}

\section{Número de Clientes Nodo NAT LANGOS AS}

\begin{tabular}{|c|c|c|c|c|c|c|c|c|c|c|}
\hline \multirow{4}{*}{ Categorías } & \multicolumn{2}{|c|}{$\begin{array}{l}\text { Ancho de } \\
\text { banda } \\
\text { asignado }\end{array}$} & \multicolumn{2}{|c|}{$\begin{array}{l}\text { PROMEDIO TOTAL } \\
\text { (paquetes recibidos) }\end{array}$} & \multicolumn{6}{|c|}{ Protocolos en \% } \\
\hline & \multirow{3}{*}{ Up } & \multirow{3}{*}{ Down } & $\%$ & $\#$ & \multirow{3}{*}{ HTTP } & \multirow{3}{*}{ HTTPS } & \multirow{3}{*}{ DNS } & \multirow{3}{*}{ SMTP } & \multirow{3}{*}{ QUIC } & \multirow{3}{*}{ QOTD } \\
\hline & & & & & & & & & & \\
\hline & & & Packet & Packet & & & & & & \\
\hline GS 2 & 2 & 2 & 15,26 & 183547 & 3,26 & 7,49 & 63,5 & 20,31 & 2,24 & 3,2 \\
\hline GS 1 & 1 & 1 & 16,09 & 207092 & 20,67 & 30,23 & 11,16 & 8,14 & 8,06 & 21,74 \\
\hline GAS 1_2 & 1 & 2 & 0 & 18 & 11,11 & 44,4 & 5,56 & 0 & 27,78 & 11,15 \\
\hline GAS 2_1 & 2 & 1 & 0,79 & 8574 & 18,66 & 40,82 & 14 & 11,66 & 12,83 & 2,03 \\
\hline GS 4 & 4 & 4 & 0 & 0 & 0 & 0 & 0 & 0 & 0 & 0 \\
\hline
\end{tabular}

Fuente: Elaboración Propia 
Si nos fijamos, en la Tabla 3, en el campo DNS para la categoría GS2 encontraremos un $63,5 \%$ que representan 116552 paquetes de un total de 600000 que se obtuvieron en promedio por captura; en la etapa de pre - procesamiento incluimos al ataque de amplificación en la categoría de tráfico sospechoso, si se relaciona esa duda con el porcentaje de DNS de la categoría GS2 hallaremos que el foco del ataque propiciado se encuentra en este grupo, esto se afirma más al observar el valor que tiene el protocolo SMTP que también posee un número elevado de paquetes y que son transmitidos por los miembros del grupo (GS2) (Díaz y Rivadeneira, 2015).

La categoría creada para el estudio GS2 contiene a 30 usuarios, en este grupo se evidencia la aparición de ataques de amplificación y de SPAM teniendo como posible causa a comprobarse la infección de algún dispositivo final perteneciente al conjunto. En esta sección se evidencia una subutilización del ancho de banda designado para la categoría GS4 mismo que en las capturas realizadas no presentó ningún paquete transmitido, quedaría por analizar si existió algún problema los días de las capturas y por ello coincidieron con la nulidad de paquetes o si se posee algún propósito predispuesto por el departamento técnico.

\section{Análisis Comparativo Escenario 2 y 3}

Se utilizarán indicadores según los requerimientos del ISP los mismos que se ven expuestos en la Tabla 4 con sus respectivas valoraciones analizadas según la metodología MIRA. Es importante indicar que se ha seguido la parametrización según el trabajo de tesis presentado por Díaz y Rivadeneira:

Tabla 4

Comparación Servidores

\begin{tabular}{ccccc}
\hline & \multicolumn{2}{c}{ HAARPCACHE } & \multicolumn{2}{c}{ RAPTORCACHE } \\
\hline INDICADORES & $\begin{array}{c}\text { VALOR } \\
\text { (1 A 10) }\end{array}$ & RESULTADO. & $\begin{array}{c}\text { VALOR } \\
\text { (1 A 10) }\end{array}$ & RESULTADO. \\
\hline Instalación & 9 & $\mathbf{0 . 9}$ & 9 & $\mathbf{0 . 9}$ \\
\hline Configuración & 9 & $\mathbf{1 . 3 5}$ & 6 & $\mathbf{0 . 9}$ \\
\hline Uso del HDD & 7 & $\mathbf{0 . 3 5}$ & 9 & $\mathbf{0 . 4 5}$ \\
\hline Uso del procesador & 10 & $\mathbf{0 . 5}$ & 9 & $\mathbf{0 . 4 5}$ \\
\hline Consumo BW local & 8 & $\mathbf{1 . 6}$ & $\mathbf{8}$ & $\mathbf{1 . 6}$ \\
\hline Cantidad de Plugins & 9 & $\mathbf{1 . 8}$ & 4 & $\mathbf{0 . 8}$ \\
\hline Solapamiento de paquetes & 10 & $\mathbf{1 . 5}$ & 1 & $\mathbf{0 . 1 5}$ \\
\hline Actualizaciones & 10 & $\mathbf{1}$ & 8 & $\mathbf{0 . 1}$ \\
\hline TOTAL: & & $\mathbf{9}$ & & $\mathbf{4 . 9}$
\end{tabular}

Fuente: Diaz y Rivadeneira (2015)

El resultado arrojado es una inclinación muy favorable para el servidor HAARPCACHE obteniendo un mejor rendimiento con un puntaje de 9 , lo que nos indica que cumple casi en su totalidad con las condiciones y requerimientos planteados por la empresa a través de los parámetros expuestos, como se puede observar de mejor manera en la figura 8. 


\section{Figura 8}

Comparativa HAARPCACHE vs RAPTORCACHE

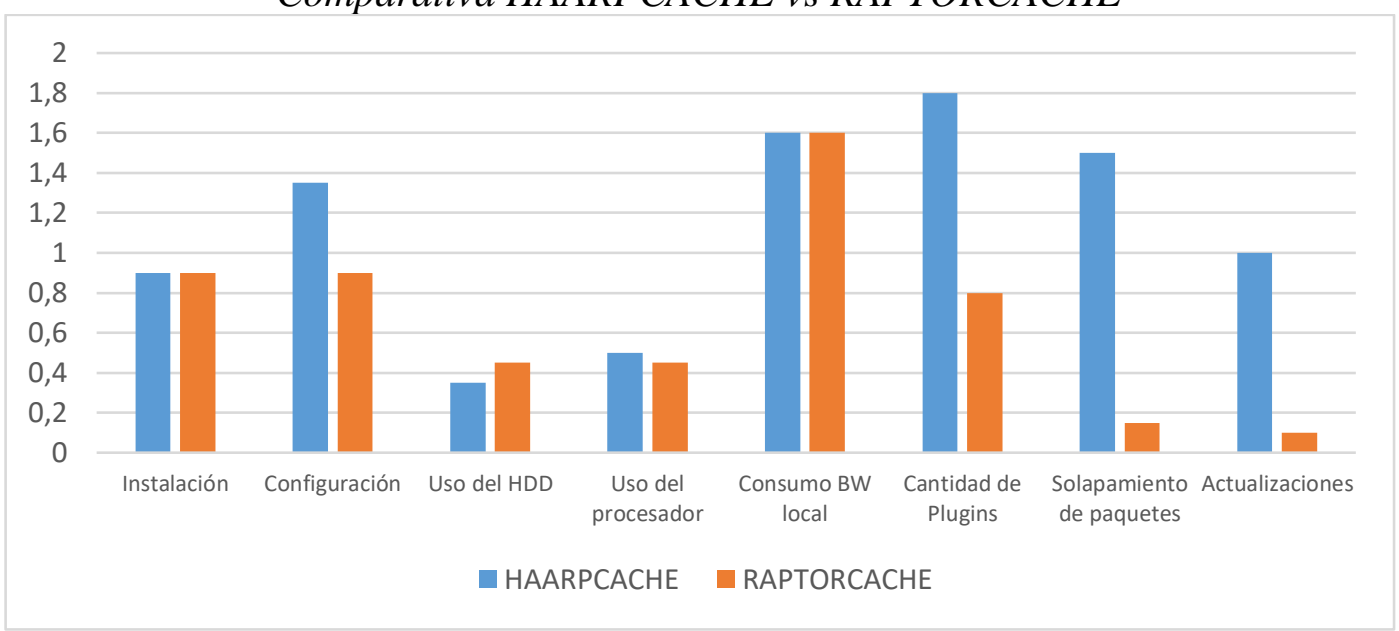

Fuente: Elaboración propia

Pero la decisión final la dará el parámetro de eficiencia del servidor para ahorrar ancho de banda, las siguientes imágenes nos permitirán tener una mejor visión y constatación de los valores este parámetro en cada escenario.

La Figura 9 representa el consumo realizado por la red Nat Langos AS durante una semana completa sin la presencia de un servidor web proxy cache, el promedio de consumo durante este período fue de 17.86 Mbps.

\section{Figura 9}

\section{Consumo Ancho de Banda Escenario 1}

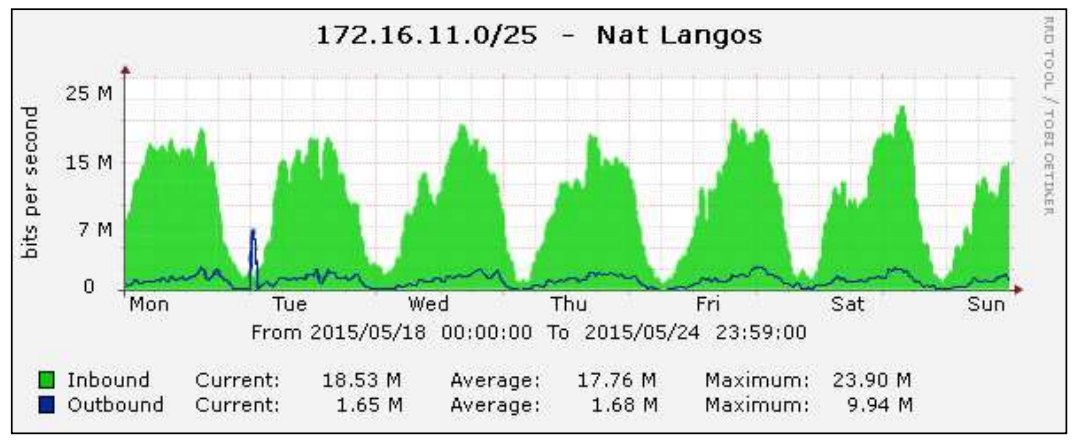

Fuente: Elaboración Propia

La Figura 10 indica el consumo de la misma red ahora con la presencia del servidor HAARPCACHE, obteniendo un promedio de $13.72 \mathrm{Mbps}$. Frente al 17.86 Mbps de consumo de ancho de banda promedio del escenario 1 el cual no contaba con ningún servidor representa un ahorro de $4.24 \mathrm{Mbps}$, un $17.35 \%$ de ahorro de los $23 \mathrm{Mbps}$ asignados a esta red. 


\section{Figura 10}

Consumo Ancho de Banda Escenario 2

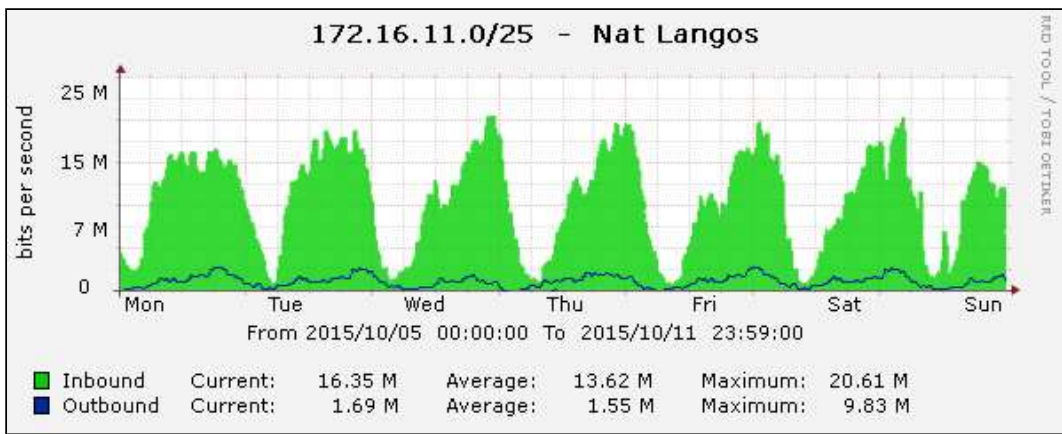

Fuente: Elaboración Propia.

Finalmente, la Figura 11 muestra el consumo realizado en el escenario 3 que poseía configurado el servidor RAPTORCACHE, el consumo promedio es reducido de 17.86 Mbps en el escenario 1 a 15.72 Mbps, un ahorro de 2.10 Mbps correspondiente a un $8.82 \%$ del total asignado a la red.

Figura 11

Consumo ancho de banda escenario 3

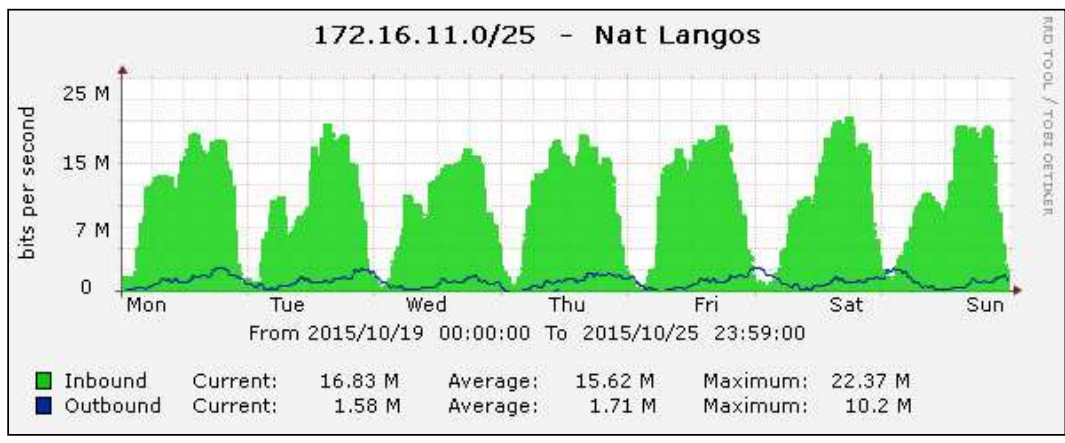

Fuente: Elaboración Propia

Tabla 6

Consumo promedio de los escenarios

\begin{tabular}{ccccccc}
\hline & \multicolumn{2}{c}{ ESCENARIO 1 } & \multicolumn{2}{c}{ ESCENARIO 2 } & \multicolumn{2}{c}{ ESCENARIO 3 } \\
\cline { 2 - 7 } Categoría & Mbps & $\%$ & Mbps & $\%$ & Mbps & $\%$ \\
\hline $\begin{array}{c}\text { Consumo Ancho de Banda } \\
\text { promedio }\end{array}$ & 17.86 & 74.2 & 13.72 & 56.8 & 15.72 & 65.8 \\
\end{tabular}

Fuente: Elaboración Propia

Podemos observar en la Tabla 6, que la implementación de un servidor web proxy cache es una buena manera para crear ese buen uso del recurso de ancho de banda en la red y en segundo lugar que el servidor de mejor prestación en este parámetro es el servidor HAARPCACHE, el cual presenta un ahorro del $17.86 \%$ del ancho de banda total asignado a la red. Por lo cual el estudio realizado sobre los escenarios indica que el servidor más apropiado para ser implementado en la red de FASTNET CIA. LTDA. debido a sus 
prestaciones y acondicionamiento a los parámetros establecidos por la empresa es el servidor HAARPCACHE.

\section{Conclusiones}

- Una vez aplicado la metodología MIRA aplicado a la red de estudio Nat Langos AS se obtuvo que los protocolos más usados son HTTP y HTTPS que equivalen al $30.92 \%$ del total de paquetes analizados y representan el $43.94 \%$ del consumo promedio de ancho de banda de la red, dichos protocolos sumado el protocolo QUIC, que figura en un $10.98 \%$ del tráfico, fueron categorizados como tráfico convencional porque su presencia corresponde a flujos de transmisión de datos comunes que utilizan páginas como facebook.com, google.com, youtube.com, entre otras.

- El tráfico sospechoso detectado en el uso de los protocolos: DNS con un $33.37 \%$ del total de paquetes, dicho valor es muy alto considerado para una red que concentra clientes tipo home evidenciando la presencia de ataques de amplificación, además se detectó la existencia de ataques de SPAM los cuales utilizan el protocolo SMTP.

- El análisis comparativo halló que el servidor HAARPCACHE cumplió en un 90\% los parámetros ideales establecidos, y, que el servidor RAPTOR CACHE cumplió con el $53.5 \%$ de dichos estamentos, el ahorro de ancho de banda empresarial provocado por el primer servidor nombrado fue del $17.86 \%$ y del segundo servidor un ahorro del $8.82 \%$. El servidor más adecuado para responder ante las necesidades de la empresa y sus condicionantes es el servidor web proxy cache HAARPCACHE.

- El servidor electo entró en actividad sin ningún problema con la red, ni con el administrador de la misma, su comportamiento es el esperado tras las pruebas mostrando un $10.78 \%$ de eficiencia durante las primeras 5 horas posteriores a su instalación

\section{Referencias bibliográficas}

Altis, K. y Luotonen, A.; (2014) World-Wide Web Proxies. Finland:Tampere University of Technology.

Cayo, S. (2015) Análisis y optimización del flujo del tráfico de protocolos y aplicaciones en la red LAN de la ESPE sede Latacunga para evaluar la calidad de servicio en base a la metodología MIRA [Tesis Publicada]. Universidad de las Fuerzas Armadas.

Costas Santos, J. (2015). Seguridad informática. RA-MA Editorial. https://elibro.net/es/lc/espoch/titulos/62452

Diaz, J. y Rivadeneira, J. (2015). Implementación de un servidor web proxy cache para reducir el consumo de ancho de banda [Tesis Publicada]. Escuela Superior Politécnica de Chimborazo. 

septiembre, 2021

Galarza, E., (2015). Configuración del proxy para el almacenamiento de las páginas más visitadas en el Instituto Tecnológico Superior Aeronáutico [Tesis Publicada]. Instituto tecnológico superior aeronáutico.

González, M., (2014), Situación de Banda Ancha en el Ecuador. [Tesis Publicada]. Universidad Politécnica Salesiana.

Haarpcache. (2018) Haarpcache web proxy. Introducción. Disponible en: http://www.haarpcache.pe

Jordan, V., (2019) Acelerando la revolución digital: Banda ancha para América Latina y el Caribe. Disponible en: http://archivo.cepal.org/pdfs/ebooks/LCR2167.pdf

Mikrocaché C. A ,(2020), Mikrocache News. Instalación de RaptorCaché. Disponible en: http://www.mikrocache.com.ve/news.html

Mikrotik, (2020), Mikrotik Documentation. Manual: Switch Chip Features. Disponible en:

http://wiki.mikrotik.com/wiki/Manual:Switch_Chip_Features\#Port_Mirroring

Montoya, C., (2019). Servidor proxy cache: comprensión y asimilación tecnológica| Dialnet. 2012. Comprensión y asimilación tecnológica. pp. 149-162 Disponible en: http://dialnet.unirioja.es/descarga/articulo/4869011.pdf

Rodríguez de Sepúlveda Maillo, D. (2015). Administración de servicios web. RA-MA Editorial. https://elibro.net/es/lc/espoch/titulos/106473

Romereal, R., (2020), MIRA: A distributed and Scalable WAN/LAN Real-time. Measurement Platform. Vol. 2511. Zurich (Switzerlamd), 2002. pp. 263-272. Disponible en: http://www.dit.upm.es/ mac/publications/2020/qofis-icqt02mira.pdf

Squid Cache Org., (2018) Squid: Optimisin Web Delivery. Optimising Web Delivery. Disponible en: http://www.squid-cache.org/

Verciana, C., (2019), MIRA: Software para el análisis de tráfico ip sobre ATM. Disponible: http://www.it.uc3m.es/azcorra/papers/2000_MIRA_Software_para_el_analisis_ de_trafico_IP_sobre_ATM.pdf

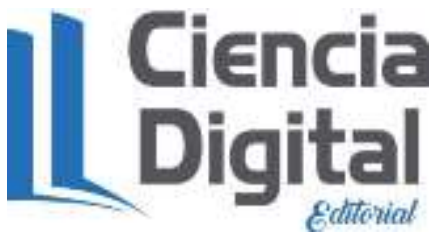




\section{PARA CITAR EL ARTÍCULO INDEXADO.}

Ramos Valencia, M. V., Salazar Cazco, S. A., Díaz León, J. G., \& Rivadeneira Romero, J. A. (2021). Análisis de la reducción del consumo de ancho de banda de un ISP mediante el estudio de Servidor Web Proxy Cache. AlfaPublicaciones, 3(3.2), 95-111. https://doi.org/10.33262/ap.v3i3.2.112

\section{LCiencia}

El artículo que se publica es de exclusiva responsabilidad de los autores y no necesariamente reflejan el pensamiento de la Revista Alfa Publicaciones.

El artículo queda en propiedad de la revista y, por tanto, su publicación parcial y/o total en otro medio tiene que ser autorizado por el director de la Revista Alfa Publicaciones.
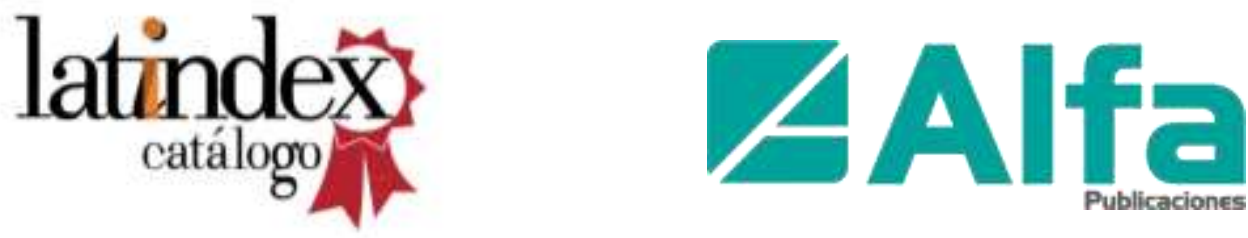